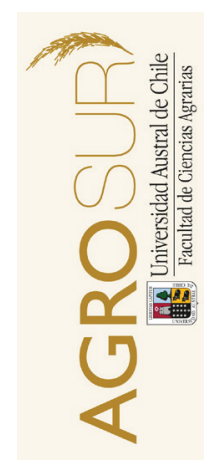

\title{
Carga ganadera y capacidad de carga de los pastizales naturales de la Patagonia chilena: estimación a nivel comunal
}

\author{
Stoking rate and grazing capacity of Chilean Patagonian rangeland: \\ Estimation at the county level \\ Castellaro, G. ${ }^{a *}$, Morales, L. ${ }^{b}$, Rodrigo, P. ${ }^{c}$ Fuentes, G. ${ }^{b}$ \\ a Departamento de Producción Animal, Facultad de Ciencias Agronómicas, Universidad de Chile. \\ Casilla 1004, Santiago, Chile. \\ b Laboratorio de Investigación en Ciencias Ambientales (LARES), Facultad de Ciencias Agronómicas, \\ Universidad de Chile. Casilla 1004, Santiago, Chile. \\ ${ }^{\mathrm{c}}$ Corporación Chileambiente. Las Abejas 5747, La Reina, Santiago.
}

\begin{tabular}{l} 
A R T I C L E I N F O \\
\hline Article history: \\
Received 03.05.2016 \\
Accepted 13.12.2016 \\
\hline Keywords: \\
Animal unit \\
Geographic information systems \\
Grazing land \\
NDVI \\
\hline Original Research Article, \\
Special Issue: Pastures for \\
Sustainable Productions Systems \\
\hline *Corresponding author: \\
Giorgio Castellaro \\
E-mail address: \\
gicastel@uchile.cl
\end{tabular}

\section{A B S T R A C T}

A study was conducted to determine the Livestock farm intensity coefficient, by the ratio between the stocking and carrying capacity of grazinglands of the Los Ríos, Los Lagos, General Carlos Ibanez del Campo (Aysén) and Magallanes and Chilean Antarctica (Chilean Patagonia) regions. The current stocking rate was determined through 2007 Livestock Census information and coverage crosses regarding pastoral rangelands at the county level, and it was compared which the carrying capacity. The latter variable was calculated from the average rangeland DM annual production, which was estimated at regional level through a simple model based on a series of 10-year-MODIS NDVI images with a pixel size of 6.25 ha approximately. Preliminary results of this analysis indicate that the rangelands of the Chilean Patagonia present high spatial variability in terms of their annual DM production. Los Lagos and Los Rios regions are those with higher stocking density, showing them the highest degree of human intervention, which in many cases results in a situation of overgrazing, contrary to what happens in many counties of the Aysén region. In this respect, the Magallanes Region presents degrees of use near the optimum, an aspect that should be taken with caution since the estimates of the model PMSA vs NDVI in shrublands and bunchgrass grassland seem to be less plausible.

\section{RESUMEN}

Se efectuó un estudio para determinar la intensidad relativa de uso de la explotación ganadera, mediante el cuociente entre la carga ganadera y la capacidad de carga de los terrenos con aptitud pastoral de las regiones de Los Ríos, Los Lagos, General Carlos Ibáñez del Campo (Aysén) y Magallanes y Antárctica Chilena (Patagonia Chilena). Mediante información del Censo ganadero del año 2007 y cruces de cobertura referidas a terrenos de pastoreo con aptitud pastoral a nivel comunal, se determinó la carga ganadera actual, la cual se comparó con la capacidad de carga. Esta última variable fue calculada a partir de la producción promedio anual de MS de los pastizales, la que se estimó a nivel regional mediante un modelo basado en una serie de 10 años de imágenes NDVI-MODIS con un tamaño de pixel de 6,25 ha aproximadamente. Los resultados preliminares de este análisis indican que los pastizales de la patagonia chilena presentan alta variabilidad espacial en cuanto a su producción anual de MS. Las regiones de Los Lagos y de Los Ríos presentan la mayor densidad animal, evidenciando en ellas el mayor grado de intervención antrópica, lo que en muchos casos se traduce en una situación de sobrepastoreo, contrariamente a lo que sucede en muchas comunas de la región de Aysén. Al respecto, la Región de Magallanes presenta grados de utilización cercana al óptimo, aspecto que debe tomarse con precaución ya que las estimaciones del modelo PMSA vs NDVI en matorrales y coironales al parecer son menos verosímiles.

Palabras clave: NDVI, terrenos de pastoreo, sistemas de información geográfica, unidad animal.

\section{INTRODUCCIÓN}

La Patagonia corresponde a la región geográfica ubicada en la parte más austral de América, que comprende territorios del sur de Argentina y Chile. En nuestro país se acepta como límite norte de esta región al sector del seno de Reloncaví y el Estrecho de Magallanes por el sur, entre el océano Pacífico y el límite con Argentina, excluyendo el archipiélago de Chiloé (Boonen, 1897). Según otros autores, el límite norte sería el río 
Calle-Calle, a los $39^{\circ}$ Lat. Sur, a la altura de Valdivia, y por el sur el Cabo de Hornos, incluyendo la Región de Aysén (Balmaceda, 1976; Campos, 2001). En gran parte de esta vasta región, una de principales actividades productivas es la ganadería extensiva, la cual se basa en la utilización de los terrenos de pastoreo con bovinos y ovinos (Covacevich y Ruz, 1996). Establecer un adecuado manejo de este recurso natural resulta relevante, ya que este no solo permite producir carne y fibra, sino que también generar una serie de servicios ecosistémicos tales como la producción de agua, el control de la erosión, el desarrollo estético en la recreación y el turismo, la producción de fauna silvestre para conservación o con propósitos cinegéticos. Todos estos aspectos contribuyen a una mejor calidad de vida de los habitantes de la región y a una mayor diversidad ecológica (Jorquera et al., 2012; Romero, 2014). En términos generales, en la zona antes delimitada, pueden diferenciarse varios tipos de pastizales naturales, entre los cuales destacan las siguientes (Ahumada y Faúndez, 2001):

\section{Praderas húmedas de tipo templado húmedo e intermedio.}

Este tipo de praderas tiene una amplia distribución geográfica, desde los sectores precordilleranos andinos de la VIII región, en tres macrozonas de la IX y X región (sector costero, valle central y precordillera) y en las regiones XI y XII donde se ubican en ecorregiones templadas húmedas e intermedias. Este gradiente les confiere diferencias dadas principalmente por la disponibilidad hídrica. En conjunto abarcan una superficie de aproximadamente 8,8 millones de ha (Ahumada y Faúndez, 2002). Ecológicamente se ubican en las provincias húmeda de verano fresco ( $\mathrm{Cfb}$ ), provincia húmeda de verano fresco y mésico ( $\mathrm{Cfsb}$ ), provincia húmeda de verano frío (Cfc) y provincia boreal húmeda invernal fría (Dfkc). Se desarrollan en una gran variedad de tipos de suelos, tales como trumaos de lomajes y cerros, trumaos planos, rojo arcillosos de lomajes, ñadis, metamórficos y de terrazas marinas (Honorato, 2000). Una característica común de estas praderas es el hecho de que siempre se desarrollan en torno a formaciones boscosas o al interior de éstos, o bien derivan de la habilitación del bosque para el pastoreo. Debido a lo anterior, existe una gran diversidad de sitios donde se desarrollan este tipo de praderas, lo cual está relacionado con variabilidad del clima, la topografía y el suelo. En estas praderas existe un dominio de plantas gramíneas rizomatosas (sobre el 50\% de la composición botánica), en especial Agrostis tenuis Sibth (sinónimo Agrostis capillaris L.) y Holcus lanatus L. También son comunes las hierbas arrocetadas y algunas leguminosas (menos de 10\% de contribución), las que en conjunto forman una pradera con aspecto de césped de vigor variable. Ocasionalmente se desarrollan al- gunas especies subarbustivas (cadillos = Acaena $s p p$ ) . En las zonas más húmedas es común la presencia de especies naturalizadas, tales como Dactylis glomerata L., H. lanatus, Loliun perenne L., Trifolium spp., Lotus uliginosus Schkur. y Lotus corniculatus L. Asociada a estas especies aparecen plantas menos deseables tales como las compuestas Leontodon sp., Taraxacum officinale L. $e$ Hypochaeris radicata L., así como también Acaena spp y $A$. tenuis. La productividad de estas praderas es muy variada, dependiendo de su ubicación ecológica, condición y niveles fertilidad en el suelo. Se citan rangos entre 500 y $14.000 \mathrm{~kg}$ de MS ha ${ }^{-1} \mathrm{año}^{-1}$. Estas productividades pueden ser modificadas por la acción antrópica mediante prácticas de mejoramiento como la fertilización (N, P, K, S), el uso de enmiendas calcáreas, la regeneración con especies cultivadas y eventualmente el drenaje y el riego (INIA, 1996). Las regiones IX y X del país, incluyen el $57,9 \%$ de la masa de bovinos y el $17,2 \%$ de la población de ovinos. A su vez, ambas regiones concentran el 73,2\% y el 61,0\% de las existencias de vacas lecheras y novillos del país, respectivamente, siendo por lo tanto, las regiones de mayor aporte a la producción de leche y carne (INE, 1997). Estos sistemas de producción son esencialmente pastoriles, siendo las praderas húmedas el principal recurso forrajero utilizados por los animales (INIA, 1996).

\section{Praderas esteparias o Coironales de la Patagonia occidental.}

Estas praderas se concentran en el sector oriental de la cordillera de los Andes de las Regiones XI de Aysén y XII de Magallanes y la Antártica Chilena (Ahumada y Faúndez, 2002), donde se presenta un clima de estepa muy fría de tendencia seco-estival (BSk'c), con precipitaciones distribuidas a lo largo del año, con montos anuales entre 100 y 400 mm (Gastó et al., 1993). En casi toda la estepa patagónica dominan suelos con relieve plano, de base formada por areniscas y arcilla, sobre los cuales se han depositado rellenos glaciales y fluvioglaciales cuaternarios, con textura generalmente franca a franco-arenosa y de profundidad media (Luzio et al., 2009). Bajo estas condiciones, se desarrolla una pradera dominada por gramíneas perennes que crecen en "champas" (géneros Festuca, Stipa, Poa y Rytiodosperma). Entre estas "champas" se desarrollan plantas en "cojín" o "inter-ciorón", con una alta diversidad de especies (poáceas de los géneros Poa, Festuca, Agrostis, Deschampsia y Phleum; hierbas dicotiledóneas, de los géneros Taraxacum, Hypochaeris, Acaena y Trifolium. Existen variaciones dadas por la pendiente (coironales planos u ondulados), ecotonos de transición con vegas, y coironales asociados a matorrales y arbustos rastreros. La productividad es muy variable, dependiendo de la precipitación, el sitio y la condición de la pradera, oscilando entre 300 y $700 \mathrm{~kg} \mathrm{ha}^{-1}$ año-1. La capacidad de carga de 
estos praderas es en promedio de 0,629 $\mathrm{EO} \mathrm{ha}^{-1} \mathrm{año}^{-1}$ (Castellaro et al., 2010). Otra formación vegetal importante en la región, lo constituyen las "vegas", las cuales corresponden a formaciones vegetales azonales, que crecen en suelos depresionales, hidromórficos, de alta fertilidad y materia orgánica, en donde se desarrolla una pradera compuesta por especies gramíneas y graminoides del alta productividad (1000-4500 kg de MS $\mathrm{ha}^{-1} \mathrm{año}^{-1}$ ). En muchos casos, éstas permanecen inundadas en gran parte del año, debido a la presencia de un horizonte impermeable, generalmente arcilloso, ubicado muy próximos a la superficie (Luzio et al., 2009). Su uso es principalmente como recurso estratégico estival.

La determinación de una correcta carga animal en los pastizales naturales antes descritos, es la más importante de todas las decisiones que involucran el manejo del pastoreo, desde el punto de vista de la vegetación, del ganado doméstico, de la fauna silvestre y de los retornos económicos (Holechek et al., 2011). A nivel de grandes extensiones de pastizales, ha sido importante la utilización de sensores remotos en la evaluación de estos ecosistemas, los cuales integrados en sistemas de información geográfica, permiten la estimación de la productividad de los pastizales, su capacidad de carga y condición (Paruelo et al., 1997; Paruelo et al., 2000; Yu et al., 2010). Sin embargo, en nuestro país y especialmente a nivel regional, escasos son los antecedentes que den cuenta de la magnitud y la variabilidad espacial que presentan los valores de capacidad de carga y de la carga ganadera actual a la cual son sometidas las praderas, especialmente en las áreas marginales, como es el caso de la Patagonia (Lara y Cruz, 1987; Pattilo et al., 2001). Por lo anterior, el objetivo de este estudio fue presentar una aproximación metodológica simple para lograr una estimación de la relación existente entre estas variables, en los diferentes tipos de pastizales naturales existentes en la Patagonia Chilena, a una escala comunal.

\section{MATERIAL Y MÉTODOS}

\section{Área del estudio}

El estudio se llevó a cabo en las comunas pertenecientes a las regiones de Los Ríos $\left(18.429,5 \mathrm{~km}^{2}\right)$, Los Lagos (48.583,5 km²), Del General Carlos Ibáñez del Campo (Aysén) $\left(108.494,5 \mathrm{~km}^{2}\right)$ y Magallanes y Antártica Chilena $\left(132.297,2 \mathrm{~km}^{2}\right)$, zona ubicada aproximadamente entre los $39^{\circ} 17^{\prime}$ y los $55^{\circ} 17^{\prime}$ de Latitud Sur, dando énfasis a los pastizales naturales presentes en ellas (Figura 1).

\section{Información cartográfica y satelital.}

Se recopiló información cartográfica del área de estudio desde bases de datos públicas en Chile, específicamente cartas regulares IGM en escala 1:50.000

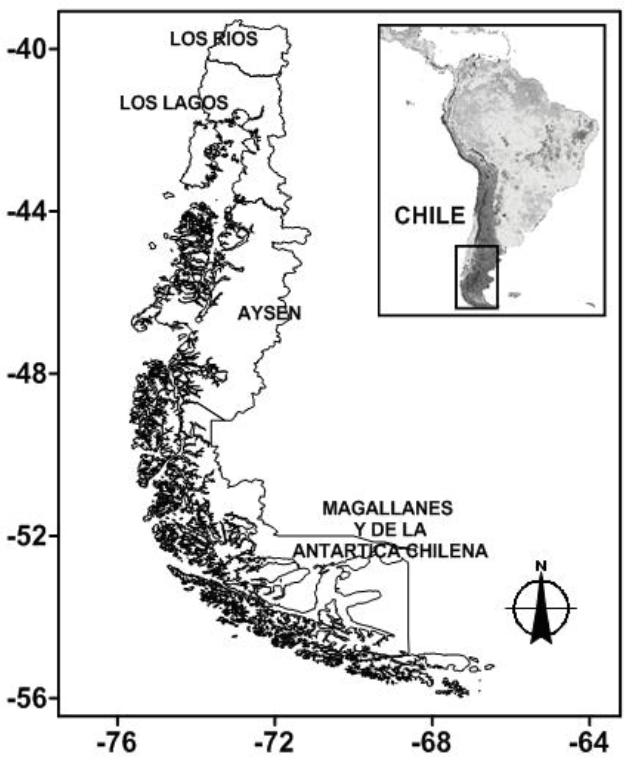

Figura 1. Área geográfica donde se realizó el estudio.

Figure 1. Geographical area where the study was conducted.

y la cartografía vegetacional del Proyecto Catastro de Bosque Nativo (CONAF, 2011; 1999). Adicionalmente, se recopiló información satelital para abordar la caracterización espacial de la vegetación utilizando imágenes de Índice de Vegetación de Diferencia Normalizada (NDVI) calculados a partir de datos provenientes de sensores remotos de libre acceso. En el presente trabajo se utilizó la serie temporal de imágenes del sensor MODIS, producto MOD13Q1 v05, que, a pesar de no tener una alta resolución espacial, se tomó en consideración por la accesibilidad de información para el área de estudio. Este producto ha sido utilizado en muchos estudios de la dinámica de la vegetación en la superficie terrestre (Morton et al., 2006; Revadekar et al., 2012; Barraza et al, 2013; Li et al., 2013; Gaitán et al., 2014) debido fundamentalmente a que consiste en imágenes de NDVI cada 16 días corregidas atmosféricamente, filtradas por sombras y nubes, e integradas por el método denominado Constraint View Maximum value composite (CV-MVC) que garantiza un ángulo de visión entre $\pm 30^{\circ}$, con una resolución espacial de $250 \mathrm{~m}$, lo que da un tamaño de píxel de aproximadamente de 6,25 ha (Ramachandran et al., 2011). Posteriormente, para contar con la condición climática media de la vegetación en el intervalo de tiempo de la serie de NDVI, se calcularon los valores medios mensuales a partir de los valores quincenales en cada mes y cada año en la serie de tiempo de 10 años de datos NDVI-MODIS (20042013). Finalmente se obtuvieron 12 imágenes correspondientes a los valores medios mensuales históricos para cada mes del año. Toda la información cartográfica e imágenes de satélite fue georreferenciada en coor- 
denadas geográficas en el Datum WGS84. El manejo y los cálculos entre coberturas fueron efectuados con el software ArcGis ${ }^{\circledR}$ cuando se trató de coberturas en formato vectorial, mientras que cuando las coberturas tuvieron formato raster, se empleó el software Idrisi ${ }^{\circledR}$.

\section{Carga ganadera actual y capacidad de carga de los pastizales.}

La Carga Ganadera (CG, UA ha ${ }^{-1}$ año ${ }^{-1}$ ) es definida como el número de unidades animales que se asignan a una superficie de pastizal por el periodo del año en el cual éste puede ser pastoreado (SRM, 1998). Por ello, la CG depende de una decisión humana. La Capacidad de Carga (CC, UA ha-1 año-1) representa la máxima carga ganadera y/o fauna silvestre que puede soportar el pastizal en forma productiva sin dar lugar que éste se deteriore. Por lo anterior, depende de factores edafoclimáticos que determinan potencialidad del sitio de pastizal, además de los objetivos y la intensidad del manejo (SRM, 1995; SRM, 1998; Holechek et al., 2011).

La CG se determinó mediante el cruce de dos coberturas. La primera de ellas dio cuenta de la población de ganado a nivel comunal, la cual fue obtenida a partir de la cantidad de animales de diferentes especies y categorías presentes en cada comuna de acuerdo al VII Censo Nacional Agropecuario y Forestal (INE, 2007). Esta información se expresó como Unidades Animales (UA), utilizando para ello los factores de equivalencia ganadera (FEG) presentados en el Cuadro 1.

Cuadro 1. Factor de equivalencia ganadera (FEG) para varios tipos de ganado doméstico.

Table 1. Livestock equivalence factor (FEG) for various types of domestic livestock.

\begin{tabular}{ll}
\hline Categoría de animal Adulto & FEG \\
\hline Bovino & 1,00 \\
Ovino & 0,20 \\
Caprino & 0,15 \\
Alpaca & 0,16 \\
Llama & 0,19 \\
Caballar & 1,25 \\
Ciervo & 0,40 \\
Asnal & 0,74 \\
Mular & 0,15 \\
\hline
\end{tabular}

Fuente: Adaptado de USDA-NRCS 2003 y calculado en base al peso metabólico de animal estándar (vaca de $450 \mathrm{~kg}$ con ternero al pie de menos de 3 meses).
Utilizando una segunda cobertura, referida a los polígonos que representan la superficie de los terrenos con aptitud pastoral que existen a nivel de cada comuna dentro de área del estudio (CONAF, 1999), se calculó para cada una de ellas, el cuociente entre las UA y dicha superficie, determinando de ese modo la carga ganadera comunal.

Para la estimación de la CC, fue necesario estimar la producción promedio anual de MS de los pastizales (PMSA, kg MS ha ${ }^{-1}$ año $^{-1}$ ), para lo cual se utilizó la ecuación propuesta por Paruelo et al. (2004), en el cual la PMSA es estimada en función del índice de vegetación normalizado (NDVI) obtenido a partir de imágenes Landsat TM. La ecuación anterior es válida para varios tipos funcionales de vegetación de la Patagonia Argentina, entre los cuales destacan formaciones boscosas, estepas arbustivas-graminosas, estepas graminosas, estepas graminosas-arbustivas, semi-desiertos y mallines:

$$
P M S A=7951,5 \cdot N D V I-1088,4 \quad[\text { Ec. } 1]
$$

El valor de NDVI utilizado fue el promedio de 10 años correspondiente al mes de diciembre, ya que este mes se considera como el más representativo de la temporada de crecimiento de los pastizales patagónicos (Paruelo et al., 2004).

A través del cruce de la cobertura que da cuenta de los polígonos que representan los diferentes tipos de terrenos de pastoreo existentes dentro de cada comuna (definidos según lo propuesto por SAG, 2003), y la imagen raster de PMSA obtenida con la Ec. [1], se calculó el valor promedio y la desviación estándar de los pixeles que representa la magnitud de PMSA, incluidos en cada polígono de vegetación con aptitud pastoral. A partir de valor promedio así obtenido, se calculó la productividad utilizable de estos terrenos, considerando la superficie que ocupa cada unidad de vegetación dentro de la comuna y el factor de uso apropiado (FUA). Esta última variable define la proporción de la MS producida en la temporada de crecimiento que puede ser consumida por el ganado, asegurando la sustentabilidad del recurso forrajero y una adecuada productividad animal. El valor del FUA depende del tipo de pastizal y puede variar entre 30 a 50\% (Holechek, 1988; Holechek et al., 2011), o incluso ser menor, cuando se trata de praderas de coironales en el cual se considera como pastoreable solamente la MS aportada por la vegetación del inter-coirón (Borrelli y Oliva, 2001). Los valores de FUA de los terrenos de pastoreo del área de estudio fueron asignados basándose en la similitud de estos con los citados por Holechek (1988) y son presentados en el Cuadro 2.

Mediante el procedimiento antes descrito, se obtuvo la Producción de MS utilizable por el ganado a nivel comunal. Al dividir este último valor por la cifra que 
Cuadro 2. Factores de uso apropiado (FUA) para los diferentes terrenos de pastoreo del área del estudio.

Table 2. Proper use factor (FUA) for the different grazing land in the study area.

\begin{tabular}{|c|c|}
\hline Tipo de terreno de pastoreo ${ }^{1}$ & FUA $(\%)^{2}$ \\
\hline Matorral Abierto & 35 \\
\hline Matorral Arborescente Abierto & 35 \\
\hline Matorral Arborescente Denso & 35 \\
\hline Matorral Arborescente Muy Abierto & 35 \\
\hline Matorral Arborescente Semi-denso & 35 \\
\hline Matorral Denso & 35 \\
\hline Matorral Muy Abierto & 35 \\
\hline Matorral Pradera Abierto & 40 \\
\hline Matorral Pradera Denso & 40 \\
\hline Matorral Pradera Semi-denso & 40 \\
\hline Matorral Semi-denso & 35 \\
\hline Ñadis Herbáceos y Arbustivos & 50 \\
\hline Praderas Perennes & 50 \\
\hline Rotación Cultivo-Pradera & 50 \\
\hline Vegas & 50 \\
\hline Estepa andina & 35 \\
\hline Estepa patagónica & 35 \\
\hline Ñadis herbáceos & 50 \\
\hline Pradera perennes & 50 \\
\hline
\end{tabular}

${ }^{1}$ Definidos según los criterios propuestos por SAG (2003) para formaciones vegetales con aptitud pastoral.

${ }^{2}$ Elaboración propia y sobre la base a Holechek (1988).

representa la demanda anual de MS de la UA (4380 kg de MS) (Scarnecchia y Kothmann, 1982; USDA-NRCS, 2003), se obtuvo la Capacidad de Carga Comunal.

Finalmente, y para evaluar la relación entre la carga ganadera y la capacidad de carga, se determinó la intensidad relativa de la explotación ganadera (Ie), calculando el cuociente entre ambas variables (Passera et al., 1986; Gastó et al., 1993):

$$
I e=\frac{C G}{C C} \quad[\text { Ec. 2] }
$$

Como la CG y la CC se expresan en las mismas unidades de medida (UA ha-1 año $^{-1}$ ), esta relación varía entre 0 (cuando un pastizal no es utilizado), hasta valores su- periores a 1 (cuando el pastizal se utiliza con una carga ganadera superior a su capacidad de sustentación lo que indica sobrepastoreo). De acuerdo con este valor relativo, se definieron clases de intensidad, según los siguientes rangos (Cuadro 3):

Cuadro 3.Valor del coeficiente que define la intensidad relativa de la explotación ganadera (Ie).

Table 3. Relative intensity livestock farm coefficient values (Ie).

\begin{tabular}{lc} 
Explotación ganadera & Valor del coeficiente Ie \\
\hline $\begin{array}{l}\text { Muy insuficiente } \\
\text { (muy extensivo) }\end{array}$ & $0,00-0,19$ \\
$\begin{array}{l}\text { Insuficiente } \\
\text { (extensivo) }\end{array}$ & $0,20-0,39$ \\
Mediano & $0,40-0,59$ \\
$\begin{array}{l}\text { Bueno } \\
\text { (intensivo) }\end{array}$ & $0,60-0,79$ \\
$\begin{array}{l}\text { Muy bueno } \\
\text { (muy intensivo, uso óptimo) }\end{array}$ & $0,80-1,00$ \\
$\begin{array}{l}\text { Sobreutilización } \\
\text { (sobrepastoreo) }\end{array}$ & $>1,00$ \\
\hline
\end{tabular}

Fuente: Passera et al. (1986).

Es importante que la CG sea, en el óptimo, cercana a la capacidad de carga teórica (Ie $\approx 1)$, de manera que se logre un pastoreo moderado (Gastó et al., 1993). En este escenario, el pastoreo permite un equilibrio entre la productividad vegetal del sistema y la presión del ganado, generando una buena dinámica y una regeneración adecuada del recurso forrajero. En la medida que la CG sea superior a la capacidad de carga (Ie > 1), se observarán efectos negativos como pérdida de cobertura vegetal, desaparición de las especies deseables (palatables), estancamiento de la propagación natural por semillas $y$, como consecuencia, incremento de la presencia de especies no deseables, lo que conlleva al sistema a una baja productividad (FAO, 1996; Holechek et al., 2011). Por otra parte, el pastoreo por debajo de la carga óptima (subpastoreo) $(\mathrm{Ie}<0,6)$, puede ocasionar el desarrollo excesivo de praderas poco productivas y la acumulación de material senescente, lo cual inhibe la productividad de la comunidad vegetal. Esta acumulación es indeseable, porque además de representar un material que no es consumido por los animales reduce la temperatura del suelo, lo que se traduce en una menor actividad bacteriana, retardando el reciclaje de nutrientes, lo que es particularmente importante en regiones frías o en épocas frías (Parton et al., 1993). 


\section{RESULTADOS Y DISCUSIÓN}

\section{Carga ganadera actual (CG)}

La carga ganadera actual (CG, UA ha ${ }^{-1}$ año $^{-1}$ ) del área bajo estudio, se presenta en la Figura 2. En las regiones de Los Ríos y de Los Lagos existen 16 comunas que presentan una CG superior a 1,0 UA ha ${ }^{-1}$ año $^{-1}$. Estas son: Curaco de Vélez, Frutillar, Futrono, Llanquihue, Los Lagos, Los Muermos, Máfil, Osorno, Paillaco, Puerto Octay, Puerto Varas, Purranque, Puyehue, Río Bueno, Río Negro y San Pablo. La CG se concentra en el valle longitudinal y en el sector oriental de la isla de Chiloé, utilizando preferentemente el tipo "Pradera perenne", el cual al ser de mayor productividad, soporta una mayor cantidad de unidades animales. En la Región del General Carlos Ibáñez del Campo, se puede observar que los sectores que particularmente exponen la mayor CG, corresponden a las comunas de Coyhaique y Río Ibáñez donde predomina el tipo vegetacional "Estepa patagónica". Por otra parte, en las comunas de Guaitecas y Tortel, se presentaron las menores CG con 0,006 y 0,003 $\mathrm{UA} \mathrm{ha}^{-1}$, respectivamente. En la Región de Magallanes y Antártica Chilena, se puede observar que en la mayoría de las comunas donde existen terrenos con aptitud pastoral, la carga ganadera es similar, lo que indicaría una distribución más homogénea de la masa ganadera, la que ocupa principalmente formaciones vegetales del tipo "Praderas perennes", "Matorral-pradera abierto" y "Estepa patagónica". Sin embargo, esta relativa mejor

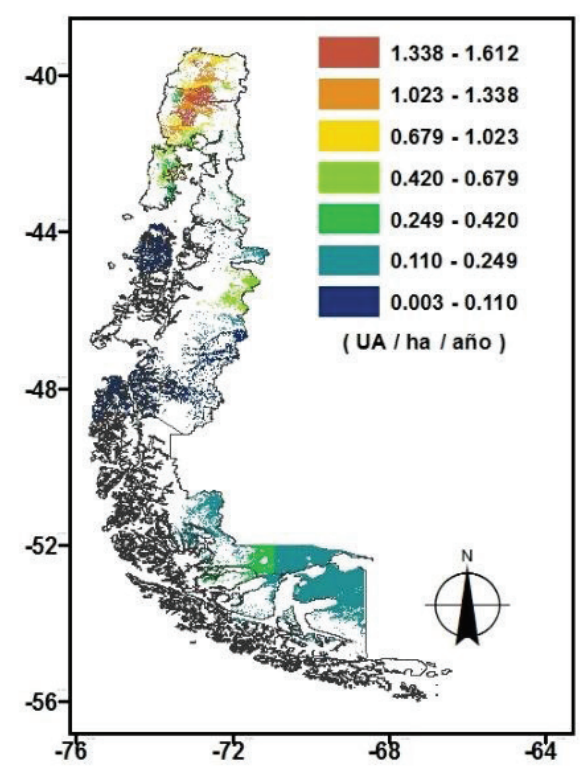

Figura 2. Carga Ganadera Actual (UA ha- ${ }^{-1}$ ño $^{-1}$ ) de los terrenos de pastoreo del área del estudio.

Figure 2. Actual stocking rate (UA ha- $\left.\mathrm{yr}^{-1}\right)$ of grazing land in the study area. distribución del ganado en esta zona, no necesariamente implica que las praderas del sector estén manejadas de acuerdo a su capacidad de sustentación.

\section{Producción Anual de Materia Seca}

En la Figura 3, se presenta la imagen que da cuenta de la producción promedio anual de MS de la región bajo estudio.

Dentro de la Regiones de Los Ríos y de Los Lagos, las comunas que presentaron la mayor producción de MS fueron Puerto Octay, Puyehue, Río Bueno y Purranque, con más de $4600 \mathrm{~kg} \mathrm{ha}^{-1} \mathrm{año}^{-1}$, correspondiendo principalmente a formaciones vegetales del tipo "Pradera perenne" (Cuadro 4). Lo anterior es consistente con la información proporcionada por Dielt y Fernández (2009), donde se señala producciones de MS cercanos a los $7000 \mathrm{~kg} \mathrm{ha}^{-1} \mathrm{anno}^{-1}$, para praderas naturalizadas en estas regiones.

En la Región del General Carlos Ibáñez del Campo, las comunas que presentan la mayor producción anual de MS fueron Los Cisnes y Guaitecas, con alrededor de $4000 \mathrm{~kg} \mathrm{ha}^{-1}$ año-1 $^{-1}$ de MS (Cuadro 5).

Las mayores productividades fueron obtenidas en formaciones vegetales del tipo "Matorral denso", "Matorral-pradera abierto" y "Matorral-pradera semidenso".

Dentro de la Región de Magallanes y Antártica Chilena, las comunas que presentaron la mayor producción anual de MS fueron Río Verde y Punta Arenas, con más de $3400 \mathrm{~kg} \mathrm{MS} \mathrm{ha}^{-1}$ año $^{-1}$ (Cuadro 6). Las mayores

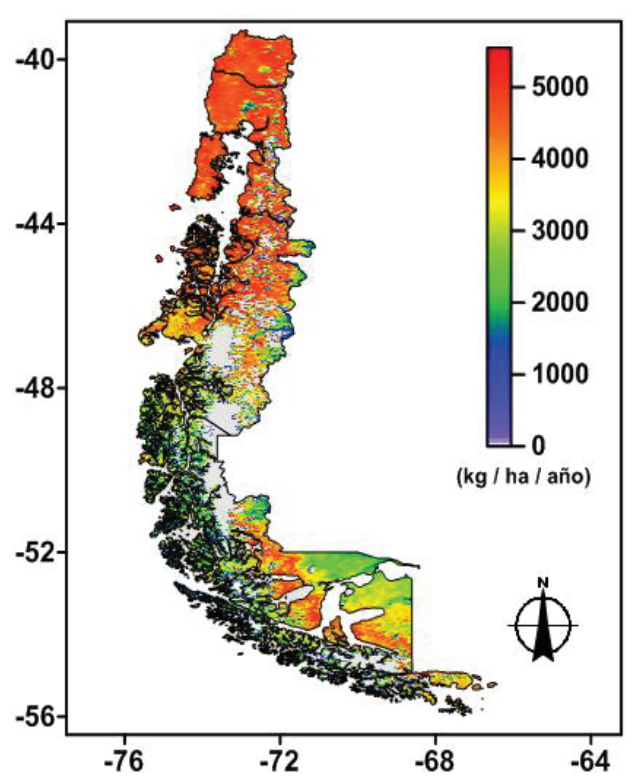

Figura 3. Producción promedio anual de materia seca $\left(\mathrm{kg} \mathrm{ha}^{-1} \mathrm{año}^{-1}\right)$ de los terrenos de pastoreo del área de estudio.

Figure 3. Average annual of dry matter production $\left(\mathrm{kg} \mathrm{ha}^{-1} \mathrm{yr}^{-1}\right)$ of grazing land in the study area. 
Cuadro 4. Producción promedio anual de materia seca (PMSA, $\mathrm{kg} \mathrm{ha}^{-1} \mathrm{año}^{-1}$ ) de los terrenos con aptitud pastoral, obtenida en las comunas de las Regiones de Los Ríos y de Los Lagos.

Table 4. Average annual of dry matter production (PMSA, $\mathrm{kg} \mathrm{ha}^{-1} \mathrm{yr}^{-1}$ ) of land with pastoral aptitude, obtained in the counties of the Los Ríos and Los Lagos Regions.

\begin{tabular}{|c|c|c|}
\hline Comuna & PMSA (kg MS ha-1 año ${ }^{-1}$ ) & Desviación estándar ( $\mathrm{kg}$ MS ha $\left.{ }^{-1} \mathrm{año}^{-1}\right)$ \\
\hline Ancud & 4134,1 & 1300,8 \\
\hline Calbuco & 3524,9 & 1534,8 \\
\hline Castro & 3181,0 & 1896,9 \\
\hline Chaitén & 3704,9 & 1781,4 \\
\hline Chonchi & 4192,2 & 1260,0 \\
\hline Cochamó & 4330,7 & 1187,7 \\
\hline Corral & 3629,2 & 1924,5 \\
\hline Curaco de Vélez & 3002,8 & 2050,5 \\
\hline Dalcahue & 4218,8 & 1231,0 \\
\hline Fresia & 4642,0 & 283,0 \\
\hline Frutillar & 4611,4 & 336,2 \\
\hline Futaleufú & 3792,8 & 1396,7 \\
\hline Futrono & 4609,7 & 467,7 \\
\hline Hualaihue & 4120,5 & 1402,0 \\
\hline La Unión & 4524,0 & 439,0 \\
\hline Lago Ranco & 4648,4 & 452,7 \\
\hline Lanco & 4642,1 & 302,4 \\
\hline Llanquihue & 4536,4 & 499,0 \\
\hline Los Lagos & 4697,9 & 381,6 \\
\hline Los Muermos & 4633,4 & 278,4 \\
\hline Máfil & 4656,8 & 246,5 \\
\hline Mariquina & 4325,9 & 1232,0 \\
\hline Maullín & 4304,4 & 749,7 \\
\hline Osorno & 4627,8 & 296,4 \\
\hline Paillaco & 4642,4 & 198,4 \\
\hline Palena & 3252,0 & 1773,4 \\
\hline Panguipulli & 4627,5 & 1300,8 \\
\hline Puerto Montt & 4020,9 & 1534,8 \\
\hline Puerto Octay & 4741,8 & 1896,9 \\
\hline Puerto Varas & 4556,8 & 1781,4 \\
\hline Puqueldón & 2992,7 & 1260,0 \\
\hline Purranque & 4660,2 & 1187,7 \\
\hline Puyehue & 4705,2 & 1924,5 \\
\hline Queilén & 2361,7 & 2050,5 \\
\hline Quellón & 2608,7 & 1231,0 \\
\hline Quemchi & 2324,4 & 283,0 \\
\hline Quinchao & 1185,1 & 336,2 \\
\hline Río Bueno & 4691,2 & 1396,7 \\
\hline Río Negro & 4625,8 & 467,7 \\
\hline San Juan de la Costa & 4305,3 & 1402,0 \\
\hline San Pablo & 4543,8 & 439,0 \\
\hline Valdivia & 3647,4 & 452,7 \\
\hline
\end{tabular}


productividades se obtuvieron en formaciones vegetales del tipo "Pradera", "Matorral denso" y "Matorralpradera abierto". No obstante lo anterior, y en especial para formaciones vegetales dominadas por matorrales, estos resultados deben tomarse con precaución, tomando en consideración que la correlación entre el NDVI y la cobertura vegetal en este tipo de formaciones vegetales es más baja en comparación a la existente en praderas herbáceas (Paruelo y Golluscio, 1994).

Otro aspecto para destacar es la alta variabilidad obtenida en los valores de producción promedio anual de MS. Según Guido et al. (2014), este aspecto es relevante ya que su conocimiento es un aspecto relevante para el manejo sustentable de sistemas de pastoreo. En general se observó una relación inversa entre la producción anual de MS y su coeficiente de variación (Cuadros 4, 5 y 6), tendiendo a ser mayor dicho coeficiente en los terrenos de pastoreo de las regiones de Aysén (44,7\% en promedio) y Magallanes y Antártica Chilena (40,3\% en promedio), en comparación con la variabilidad de la producción de MS obtenida en las regiones de Los Ríos y Los Lagos, donde esta variabilidad fue en promedio del orden del $28 \%$. Los resultados anteriores confirman la tendencia que indica que la productividad de los pastizales de la región patagónica es altamente variable del punto de vista espacial, lo que se acentúa

Cuadro 5. Producción promedio anual de materia seca (PMSA, $\mathrm{kg} \mathrm{ha}^{-1} \mathrm{año}^{-1}$ ) de los terrenos con aptitud pastoral, obtenida en las comunas de la Región del General Carlos Ibáñez del Campo.

Table 5. Average annual of dry matter production (PMSA, $\mathrm{kg} \mathrm{ha}^{-1} \mathrm{yr}^{-1}$ ) of land with pastoral aptitude, obtained in the counties of General Carlos Ibáñez del Campo Region.

\begin{tabular}{|c|c|c|}
\hline Comuna & PMSA (kg MS ha-1 año $^{-1}$ ) & Desviación estándar ( $\mathrm{kg}$ MS ha-1 $\left.\mathrm{año}^{-1}\right)$ \\
\hline Aysén & 3865,3 & 1386,0 \\
\hline Chile Chico & 2014,3 & 906,4 \\
\hline Cisnes & 4210,2 & 1604,8 \\
\hline Cochrane & 2729,8 & 900,1 \\
\hline Coihaique & 3378,5 & 1625,4 \\
\hline Guaitecas & 3977,9 & 1300,7 \\
\hline Lago Verde & 2792,9 & 762,9 \\
\hline Río Ibáñez & 2587,7 & 1510,0 \\
\hline Tortel & 2841,9 & 1551,6 \\
\hline O'Higgins & 1815,6 & 1335,2 \\
\hline
\end{tabular}

Cuadro 6. Producción promedio anual de materia seca (PMSA, $\mathrm{kg} \mathrm{ha}^{-1} \mathrm{año}^{-1}$ ) de los terrenos con aptitud pastoral, obtenida en las comunas de la Región de Magallanes y Antártica Chilena.

Table 6. Average annual of dry matter production (PMSA, $\mathrm{kg} \mathrm{ha}^{-1} \mathrm{yr}^{-1}$ ) of land with pastoral aptitude, obtained in the counties of Magallanes and Antártica Chilena Region.

\begin{tabular}{lcc}
\hline Comuna & PMSA (kg MS ha $\mathbf{~ a n ̃ o ~}^{-\mathbf{1}}$ ) & Desviación estándar $\mathbf{~ k g ~}$ MS ha $^{-\mathbf{1}}$ año $^{-\mathbf{1}}$ ) \\
\hline Cabo de Hornos & 2178,7 & 1393,0 \\
Laguna Blanca & 2882,1 & 1761,1 \\
Natales & 3040,5 & 670,2 \\
Porvenir & 3122,2 & 1624,8 \\
Primavera & 2966,7 & 782,1 \\
Punta Arenas & $\mathbf{3 3 7 6 , 2}$ & 338,6 \\
Río Verde & $\mathbf{3 4 5 5 , 5}$ & 1162,4 \\
San Gregorio & 2353,1 & 1244,7 \\
Timaukel & 3219,4 & 673,9 \\
Torres del Paine & 2254,3 & 1343,4 \\
\hline
\end{tabular}


en zonas con menores montos pluviométricos. Similar tendencia en la variabilidad de la productividad de praderas a escala regional son reportados por Jia et al., (2015) en praderas templadas de China. Además de aspectos evidentes atribuidos a la diferente estructura de la vegetación, dicha variación estaría asociada fundamentalmente a diferencias en características de los suelos, en especial la profundidad y a variaciones en la disponibilidad hídrica entre diferentes unidades geomorfológicas (Guido et al., 2014).

\section{Capacidad de carga de los pastizales}

La capacidad de carga de los terrenos de pastoreo de la región bajo estudio, se presenta en la Figura 4. En las Regiones de Los Ríos y de Los Lagos, los mayores valores de capacidad de carga, se obtuvieron en las comunas de Frutillar, Llanquihue y Río Bueno, donde dominan las formaciones vegetales del tipo "Praderas perennes", los cuales son los terrenos de pastoreo de mayor productividad. Esta tendencia es de esperar, ya que la capacidad de carga es una función directa de dicha productividad.

En la Región del General Carlos Ibáñez del Campo, y al igual de lo ocurrido en las regiones de Los Lagos y de Los Ríos, las mayores capacidades de carga se obtuvieron en los sectores donde hay predominio de formaciones vegetales del tipo "Praderas perennes", así como en el tipo “Estepa patagónica”, las cuales presentan la mayor

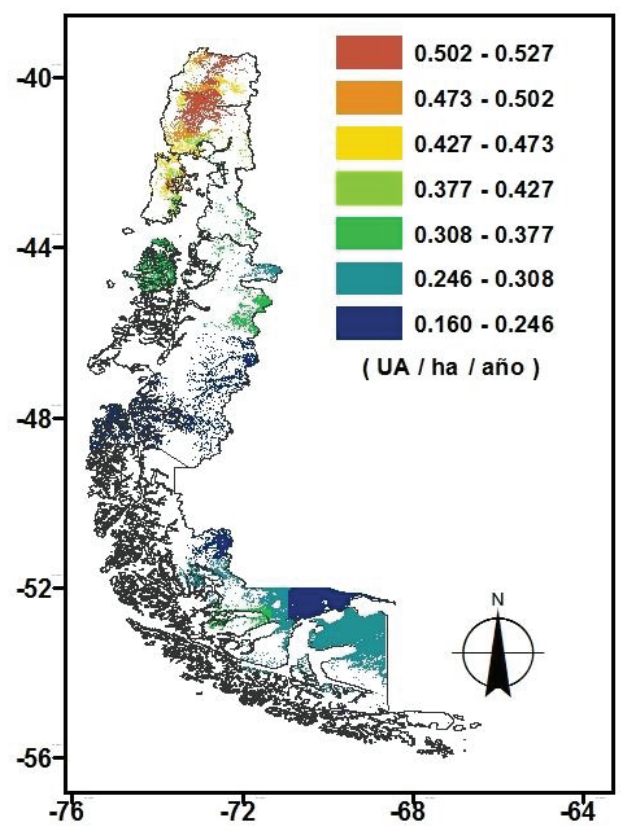

Figura 4. Capacidad de carga (UA ha ${ }^{-1}$ año $^{-1}$ ) de los terrenos de pastoreo de del área del estudio.

Figure 4. Grazing Capacity (UA ha ${ }^{-1} \mathrm{yr}^{-1}$ ) of grazing land in the study area. productividad. No obstante lo anterior, el rango de variación obtenido en esta variable fue de menor magnitud en comparación al determinado en las regiones X y XIV.

En la Región de Magallanes y Antártica Chilena, se obtuvieron capacidades de carga que caen dentro de un rango de variación similar al obtenido en la XI Región del General Carlos Ibáñez del Campo, pero con un predominio de valores que varía entre 0,2 y $0,3 \mathrm{UA} \mathrm{ha}^{-1}$ $\mathrm{año}^{-1}$. Esta cifras equivalen a mantener en una ha de terreno 1,0 a 1,5 ovinos, valores que pueden ser adecuados para pastizales esteparios manejados con cargas medias en donde se observa disminución de coirones y aumento de pastos cortos (Covacevich, 2012). Sin embargo, otras estimaciones puntuales, indican que estas cifras podrían ser considerada excesivas (SAG, 2003; Castellaro et al., 2010).

Como una forma de mejorar las estimaciones efectuadas por el presente modelo, debiesen ser considerados reducciones en la capacidad de carga atribuidos a factores como la distancia a las fuentes de agua, grado de pendiente y erosión de suelos (Yu et al., 2010).

\section{Intensidad Relativa de Explotación Ganadera}

La Figura 5 muestra los valores obtenidos en el coeficiente intensidad relativa de explotación ganadera (Ie) de las regiones bajo estudio.

En la mayoría de las comunas de las regiones de Los Ríos y Los Lagos, los valores de este indicador fueron

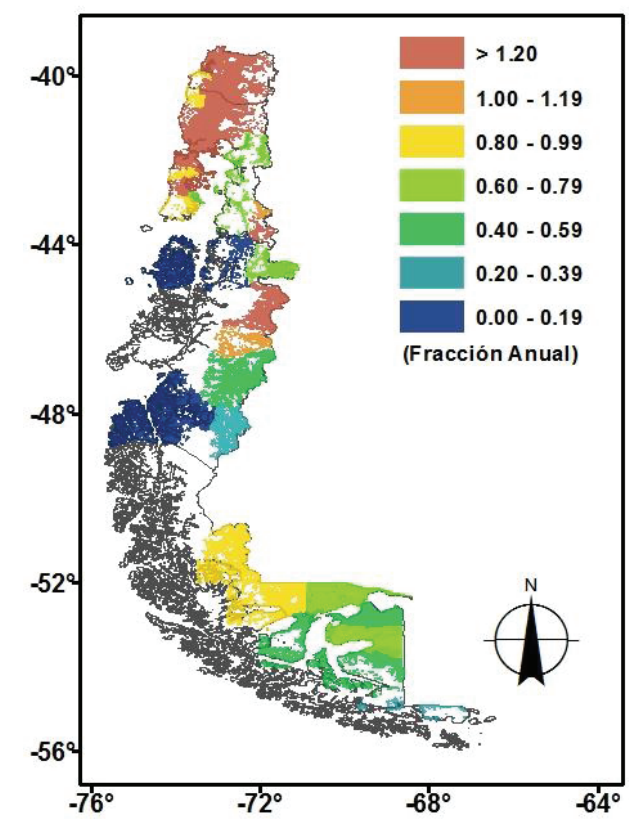

Figura 5. Coeficiente de intensidad de explotación ganadera (Ie fracción anual) de los terrenos de pastoreo del área del estudio.

Figure 5. Livestock farm intensity coefficient (Ie, annual fraction) for grazing land in the study area. 
mayores a 1,0, lo que indica, que desde el punto de vista de la utilización de los terrenos de pastoreo, existiría una situación de sobre-pastoreo. Se excluyen de esta generalidad las comunas de Chaitén, Cochamó, Corral, Dalcahue, Hualaihue, las que observan un valor de Ie que indica una situación de intensidad de la explotación ganadera clasificada como buena a muy buena. Las cifras obtenidas por este indicador en cada comuna de estas regiones se presentan en el Cuadro 7.

De acuerdo a los valores expuestos en el Cuadro 7, en gran parte de las comunas de esta región sería recomendable la disminución de la carga ganadera. Las cifras anteriores denotan además, una alta intervención antrópica de los pastizales naturales.

En la Región de General Carlos Ibáñez del Campo, las variaciones obtenidas en este indicador fueron de menor magnitud a obtenido en las regiones X y XIV (Cuadro 8).

Los valores de Ie, en general fueron inferiores a 0,6 , lo que indicaría una situación de subutilización de los terrenos con aptitud pastoral. La excepción a esta norma lo constituyen las comunas de Coyhaique y Río Ibáñez, donde se confirma una sobreutilización del recurso pastoril. De acuerdo con las cifras anteriores, en algunos sectores de esta región se podría incrementar la masa ganadera en forma significativa, pero considerando un grado de intervención humana moderado a bajo. La excepción a esta regla serían las comunas de Coyhaique y Río Ibáñez, donde lo recomendable sería disminuir la carga.

Los sistemas ganaderos de la Región de Magallanes y Antártica Chilena presentan un uso que está dentro de los rangos clasificados como en medianos a muy buenos, con la excepción de la comuna de Cabo de Hornos, donde el grado de intensidad de la explotación ganadera es considerado como insuficiente (Cuadro 9).

De acuerdo con las cifras anteriores, en esta región se recomienda no aumentar la masa ganadera, dado que existiría un equilibrio entre la productividad de los pastizales y la demanda de MS de los animales que los pastorean. Las cifras también sugieren un grado de intervención moderado a alto de los terrenos de pastoreo. No obstante, y en especial en pastizales de matorrales y coironales esteparios de esta región, mediciones de terreno (Covacevich y Ruz, 1996; SAG, 2003) y evaluaciones de erosión de suelos (CIREN, 2010) indican un alto grado de deterioro del recurso pastoril, lo que podría poner en duda las estimaciones realizadas con este modelo en dicha situación.

\section{CONCLUSIONES}

Los sistemas ganaderos de la Patagonia Chilena, se basan principalmente en la utilización de las praderas mediante el pastoreo directo y en forma extensiva, donde la productividad de los mismos depende de cuan efi-
Cuadro 7. Valores del coeficiente de intensidad de explotación ganadera (Ie) obtenido en los terrenos de pastoreo de las regiones de Los Lagos y de Los Ríos.

Table 7. Livestock farm intensity Coefficient values (Ie) obtained in the grazing lands of Los Lagos and Los Rios Regions.

\begin{tabular}{|c|c|c|}
\hline Comuna & IE & $\begin{array}{c}\text { Grado de la Explotación } \\
\text { Ganadera }\end{array}$ \\
\hline Ancud & 1,38 & Sobreutilización \\
\hline Calbuco & 1,43 & Sobreutilización \\
\hline Castro & 1,42 & Sobreutilización \\
\hline Chaitén & 0,72 & Bueno \\
\hline Chonchi & 1,20 & Sobreutilización \\
\hline Cochamó & 0,65 & Bueno \\
\hline Corral & 0,84 & Muy Bueno \\
\hline Curaco de Vélez & 2,46 & Sobreutilización \\
\hline Dalcahue & 0,81 & Muy Bueno \\
\hline Fresia & 1,74 & Sobreutilización \\
\hline Frutillar & 2,81 & Sobreutilización \\
\hline Futaleufú & 1,01 & Sobreutilización \\
\hline Futrono & 2,57 & Sobreutilización \\
\hline Hualaihue & 0,71 & Bueno \\
\hline La Unión & 1,63 & Sobreutilización \\
\hline Lago Ranco & 1,43 & Sobreutilización \\
\hline Lanco & 1,77 & Sobreutilización \\
\hline Llanquihue & 2,90 & Sobreutilización \\
\hline Los Lagos & 2,38 & Sobreutilización \\
\hline Los Muermos & 2,36 & Sobreutilización \\
\hline Máfil & 2,72 & Sobreutilización \\
\hline Mariquina & 1,53 & Sobreutilización \\
\hline Maullín & 1,83 & Sobreutilización \\
\hline Osorno & 2,70 & Sobreutilización \\
\hline Paillaco & 2,56 & Sobreutilización \\
\hline Palena & 1,22 & Sobreutilización \\
\hline Panguipulli & 1,54 & Sobreutilización \\
\hline Puerto Montt & 1,20 & Sobreutilización \\
\hline Puerto Octay & 2,60 & Sobreutilización \\
\hline Puerto Varas & 2,11 & Sobreutilización \\
\hline Puqueldón & 1,67 & Sobreutilización \\
\hline Purranque & 2,91 & Sobreutilización \\
\hline Puyehue & 2,97 & Sobreutilización \\
\hline Queilén & 0,73 & Bueno \\
\hline Quellón & 0,94 & Muy bueno \\
\hline Quemchi & 1,24 & Sobreutilización \\
\hline Quinchao & 1,74 & Sobreutilización \\
\hline Río Bueno & 3,07 & Sobreutilización \\
\hline Río Negro & 2,56 & Sobreutilización \\
\hline San Juan de la Costa & 0,81 & Muy bueno \\
\hline San Pablo & 2,30 & Sobreutilización \\
\hline Valdivia & 1,60 & Sobreutilización \\
\hline
\end{tabular}


Cuadro 8. Valores del coeficiente de intensidad de explotación ganadera (Ie) obtenido en los terrenos de pastoreo de la región del General Carlos Ibáñez de Campo.

Table 8. Livestock farm intensity Coefficient values (Ie) obtained in the grazing lands of General Carlos Ibáñez de Campo Region.

\begin{tabular}{lcc}
\hline Comuna & IE & $\begin{array}{c}\text { Grado de la Explotación } \\
\text { Ganadera }\end{array}$ \\
\hline Aysén & 0,46 & Mediano \\
Chile Chico & 0,57 & Mediano \\
Cisnes & 0,15 & Muy insuficiente \\
Cochrane & 0,46 & Mediano \\
Coyhaique & 1,49 & Sobreutilización \\
Guaitecas & 0,02 & Muy insuficiente \\
Lago verde & 0,70 & Bueno \\
Río Ibáñez & 1,04 & Sobreutilización \\
Tortel & 0,01 & Muy insuficiente \\
O’Higgins & 0,20 & Muy Insuficiente \\
\hline
\end{tabular}

ciente se utilice el pastizal, especialmente en aspectos relacionados con el ajuste de la carga ganadera. Dicha productividad es altamente variable del punto de vista espacial, lo que se acentúa en zonas con menores montos pluviométricos.

Los pastizales de las regiones de Los Lagos y de Los Ríos son las que presentan mayor densidad animal, evidenciando en ellas el mayor grado de intervención antrópica, lo que en muchos casos se traduce en una situación de sobreutilización. En varias comunas de estas regiones debiera intervenirse el manejo fundamentalmente orientado a una disminución de la carga ganadera.

Contrariamente a lo anterior, y dado el nivel de productividad actual de los terrenos de pastoreo, la Región de General Carlos Ibáñez del Campo (Aysén) es la presenta los menores grados de utilización del punto de vista ganadero. En la mayoría de las comunas de esta Región se podría incrementar la masa de ganado.

De acuerdo a los resultados obtenidos con el modelo utilizado en este trabajo, la Región de Magallanes presentaría grados de utilización cercana al óptimo. Lo anterior debe tomarse con precaución, dado que es muy importante validar el método de la estimación de la producción promedio anual de MS en condiciones locales y en una escala de mayor resolución, especialmente en terrenos de pastoreo dominados por formaciones vegetales de matorrales y coironales, donde la correlación entre el NDVI y la producción de MS aérea es más débil.
Cuadro 9. Valores del coeficiente de intensidad de explotación ganadera (Ie) obtenido en los terrenos de pastoreo de la región de Magallanes y Antártica Chilena.

Table 9. Livestock farm intensity Coefficient values (Ie) obtained in the grazing lands of Magallanes and Antártica Chilena Region.

\begin{tabular}{lcc}
\hline \multicolumn{1}{c}{ COMUNA } & Ie & $\begin{array}{c}\text { Grado de Explotación } \\
\text { Ganadera }\end{array}$ \\
\hline Cabo de Hornos & 0,25 & Insuficiente \\
Laguna Blanca & 0,90 & Muy Bueno \\
Natales & 0,88 & Muy bueno \\
Porvenir & 0,72 & Bueno \\
Primavera & 0,53 & Mediano \\
Punta Arenas & 0,55 & Mediano \\
Río Verde & 0,90 & Muy Bueno \\
San Gregorio & 0,61 & Bueno \\
Timaukel & 0,58 & Mediano \\
Torres del Paine & 0,80 & Muy bueno \\
\hline
\end{tabular}

\section{Agradecimientos}

El presente artículo fue financiado por el Ministerio de Bienes Nacionales, Chile, en el marco del proyecto "La Patagonia Chilena y una propuesta de criterios para su desarrollo sustentable" durante los años 2012 y 2013.

\section{REFERENCIAS}

Ahumada, M.C., Faúndez, L.Y., 2002. Guía descriptiva de las praderas naturales de Chile. Ministerio de Agricultura, Servicio Agrícola y Ganadero, Departamento de Protección de los Recursos Naturales Renovables. Santiago.

Balmaceda, R. R., 1976. Geografía Histórica de la Patagonia. Ediciones Cervantes. Santiago, Chile.

Barraza, V., Grings, F., Salvia, M., Perna, P., Carbajo, A. E., Karszenbaum, H., 2013. Monitoring and modelling land surface dynamics in Bermejo River Basin, Argentina: time series analysis of MODIS NDVI data. International Journal of Remote Sensing 34(15), 5429-5451.

Boonen, J., 1897. Ensayo sobre la geografía militar de Chile. Volumen 2. Imprenta Cervantes. Santiago, Chile.

Borrelli, P., Oliva, G., 2001. Evaluación de pastizales, in: Borrelli, P., Oliva, G. (Eds.), Ganadería Sustentable en la Patagonia Austral. INTA Reg. Pat. Sur, pp. 161-182.

Campos, M. E., 2002. El alma de la Patagonia. Editorial Larivière, Buenos Aires.

Castellaro, G., Morales, L., Ahumada, M., Barozzi, A., 2010. Simulation of dry matter productivity and water dynamics in a Chilean Patagonian range. Chilean Journal of Agricultural Research 70(3), 417-427.

Centro de Información de Recursos Naturales (CIREN), 2010. 
Determinación de Erosión Potencial y actual de Chile. Código: 06CN12IAM-12. Informe Técnico Final. Formato INNOVA-CHILE. Santiago, Chile.

Corporación Nacional Forestal (CONAF)., 2011. Catastro de los Recursos Vegetacionales Nativos de Chile. Monitoreo de cambios y actualizaciones. Período 1997-2011. Corporación Nacional Forestal. Santiago, Chile.

Corporación Nacional Forestal (CONAF), 1999. Catastro y evaluación de recursos vegetacionales nativos de Chile. Proyecto CONAF-CONAMA-BIRF Universidad Austral de Chile. Pontificia Universidad Católica de Chile. Universidad Católica de Temuco. 89 p. Santiago.

Covacevich, N., Ruz, J., 1996. Praderas en la zona austral: XII Región (Magallanes). Capítulo 37, in: Ruiz, N.I. (Ed.) Praderas para Chile. $2^{\underline{a}}$ ed. Ministerio de Agricultura, Instituto de Investigaciones Agropecuarias (INIA), Santiago. pp. 639-655.

Covacevich, C. N., 2012. Sistemas de pastoreo en Magallanes. Capítulo 7, in: Strauch, B. O., Lira, F. R. (Eds.), Bases para la Producción Ovina en Magallanes. Instituto de Investigaciones Agropecuarias. Boletín INIA N² 244. pp. 104114.

Dietl, W., Fernández, F., 2009. Manejo sostenible de praderas. Su flora y vegetación. Boletín INIA № 187. Instituto de Investigaciones Agropecuarias, Cauquenes, Chile.

Gaitán J., Bran, D., Oliva, G., Maestre, F. T., Aguiar, M. R., Jobbágy, E., Buono, G., Ferrante, D., Nakamatsu, V., Ciari, G., Salomone, J. Massara, V., 2014. Plant species richness and shrub cover attenuate drought effects on ecosystem functioning across Patagonian rangelands. Biol. Lett. 10, 20140673. doi: 10.1098/rsbl.2014.0673.

Gastó J., Cosio, F., Panario D., 1993. Clasificación de Ecorregiones y determinación de sitio y condición. Manual de Aplicación a municipios y predios rurales. Red de Pastizales Andinos. Santiago, Chile.

Guido, A., Díaz, R., Baldassini, P., Paruelo, J., 2014. Spatial and Temporal Variability in Aboveground Net Primary Production of Uruguayan Grasslands. Rangeland Ecology and Management 67, 30-38.

Holben, B., 1986. Characteristics of Maximum-Value composite image from temporal AVHRR data. International Journal of Remote Sensing, 1417-1464.

Holechek J. L., 1988. An approach for setting the stoking rate, Rangelands 10, 10-14.

Holechek, J.L., Pieper, R.D., Herbel, C.H., 2011. Range Management, Principles and Practices. $6^{\text {th }}$ edition. Prentice Hall, New Jersey.

Honorato, P. R., 2000. Manual de Edafología. 4a Edición. Alfaomega. Ediciones Universidad Católica de Chile., Santiago.

Instituto de Investigaciones Agropecuarias (INIA), 1996. Praderas para Chile. 2 ${ }^{a}$ Edición. Instituto de Investigaciones Agropecuarias, Ministerio de Agricultura, Santiago.

Instituto Nacional de Estadísticas (INE), 2007. VII Censo Nacional Agropecuario. 1997. Total Nacional. Santiago.

Jia X., Xie, B., Shao M., Zhao, C., 2015. Primary Productivity and Precipitation-Use Efficiency in Temperate Grassland in the Loess Plateau of China. PLoS ONE 10(8), e0135490. doi:10.1371/journal.pone.0135490.

Jorquera-Jaramillo, C., Alonso, J. M., Aburto, J., Martínez-Tillería, K., León, M. F., Pérez, A., Gaymer, C. F., Squeo, F. A.,
2012. Conservación de la biodiversidad en Chile: Nuevos desafíos y oportunidades en ecosistemas terrestres y marinos costeros. Revista Chilena de Historia Natural, 85(3), 267-280.

Lara, A., Cruz, G., 1987. Evaluación de potencial de pastoreo del área de uso agropecuario de la XII Región, Magallanes y de la Antártica Chilena. INIA-Intendencia XII Región. Punta Arenas, Chile.

Li, S., Xie, Y., Brown, D.G., Bai, Y., Hua, J., Judd, K., 2013. Spatial variability of the adaptation of grassland vegetation to climatic change in Inner Mongolia of China. Applied Geography 43, 1-12.

Luzio W., Casanova, M., Seguel, O., 2009. Suelos de la Zona de Magallanes (Desde $50^{\circ} \mathrm{LS}$ hasta $55^{\circ} \mathrm{LS}$ ), in: Luzio, W. (Ed.), Suelos de Chile. Universidad de Chile. Santiago, pp. 291-306.

Morton, D. C., DeFries, R. S., Shimabukuro, Y. E., Anderson, L. O., Arais, E., del Bon Espirito-Santo, F., Freitas, R., Morisette, J., 2006. Cropland expansion changes deforestation dynamics in the southern Brazilian Amazon. PNAS 103(39), 14637-14641.

Organización de las Naciones Unidas para la Agricultura y la Alimentación (FAO), 1996. Principios de manejo de praderas naturales. $2^{\circ}$ Ed. Oficina Regional de la FAO para América Latina y el Caribe. Santiago, Chile.

Parton, W. J., Scurlock, M. O., Ojima D. S., Gilmanov, T. G., Scholes, R. J., Schimel, D. S., Kirchner, T., Meneaut, J. C., Seastedt, T., García-Moya, E., Kamnalrut, A., Kinyamario, J. I., 1993. Observations and modelling of biomass and soil organic matter dynamics for the grassland biome worldwide. Global Biogeochemical Cycles 7(4), 785 - 809.

Paruelo J. M., Goluscio, R. A., 1994. Range assessment using remote sensing in Northwest Patagonia (Argentina). Journal of Range Management 47(6), 498-502.

Paruelo, J.M., Epstein, H. E., Lauenroth, W. K., Burke, I.C., 1997. ANPP estimates from NDVI for the central grassland region of the United States. Ecology 78(3), 953-958.

Paruelo, J. M., Oesterheld, M., Di Bella, C. M., Arzadum, M., Lafontaine, J., Cahuepé, M., Rebella, C. M., 2000. Estimation of primary production of subhumid rangelands from remote sensing data. Applied Vegetation Science 3, 189195.

Paruelo J., M., Golluscio, R. A., Guersschman, J. P., Cesa, A., Jouve V. V., Garbulsky, M. F., 2004. Regional scale relationships between structure and functioning. The case of the Patagonian steppes. Global Ecology and Biogeography 13 (5), 385-395.

Passera, C. B., Dalmasso, A. D., Borsetto, O., 1986. Método de "Point Quadrat Modificado", in: Subcomité Asesor del Árido Subtropical Argentino (Ed.), Taller de Arbustos forrajeros para zonas áridas y semiáridas $2^{\text {da }} \mathrm{Ed}$. Buenos Aires, pp. 71-79.

Pattilo, C., Covacevich, N., Pezoa, M. E., Mardones, L., 2001. Aplicación de teledetección y SIG en la caracterización de la capacidad ganadera ovina de los predios de la comuna de laguna Blanca, XII Región de Magallanes y la Antártica Chilena, in: Strauch, O., Covacevich, N., Cárdenas, A., (Eds.), Manejo sustentable de las praderas naturales de Magallanes. SOCHIPA en Magallanes 30 años. Instituto de Investigaciones Agropecuarias (INIA). Serie actas INIA N 35 ., Punta Arenas, pp. 86-87. 
Ramachandran, B., Christopher, J. O., Michael, A. J., 2011. Land Remote Sensing and Global Environmental Change: NASA's Earth Observing System and the Science of ASTER and MODIS. Springer, New York.

Revadekar, J.V., Tiwari, Y.K., Kumar, K.R., 2012. Impact of climate variability on NDVI over the Indian region during 1981-2010. International Journal of Remote Sensing 33, 7132-7150.

Romero, H., 2014. Ecología política y represas: elementos para el análisis del Proyecto HidroAysén en la Patagonia Chilena. Revista de Geografía Norte Grande 57, 161-175.

Scarnecchia, D. L., Kothmann, M.M., 1982. A Dynamic approach to grazing management terminology. Journal of Range Management 35(2), 262-264.

Servicio Agrícola y Ganadero (SAG). 2003. El Pastizal de Tierra del Fuego. Guía de uso, condición actual y propuesta de seguimiento para determinación de tendencia.
Gobierno Regional de Magallanes y Antártica Chilena. Ministerio de Agricultura. Servicio agrícola y Ganadero (SAG). Departamento de Protección de los Recursos Naturales Renovables (DEPROREN). Punta Arenas, Chile.

Society for Range Management (SRM), 1995. New concepts for assessment of rangeland condition. Journal of Range Management 48(3), 271-282.

Society for Range Management (SRM), 1998. Glossary of terms used in range management, $4^{\text {th }}$ Ed. Denver, CO.

United States (USDA) - Natural Resources Conservation Service (NRCS). 2003. National Range and Pasture Handbook, USDA Natural Resources Conservation Service Grazing Lands Technology Institute. Cap. 6. Fort Worth, Texas.

Yu, L., Zhou, L., Liu, W., Zhou, H. K., 2010. Using remote sensing and GIS technologies to estimate grass yield and livestock carrying capacity of alpine grasslands in Golog Prefecture, China. Pedosphere 20(3), 342-351. 
